\title{
An Off-Axis Electron Holography Study of the Built-In Electric Field in $\mathrm{LaAlO}_{3} / \mathrm{SrTiO}_{3}$ Heterointerface
}

\author{
Lin $\mathrm{XIE}^{1, \mathrm{a},{ }^{*}}$ \\ ${ }^{1}$ National Laboratory of Solid State Microstructures and College of Engineering and Applied Sciences, \\ Nanjing University, Nanjing, Jiangsu 210093, People's Republic of China \\ axielin@nju.edu.cn \\ ${ }^{*}$ Corresponding author
}

Keywords: Electron holography, $\mathrm{LaAlO}_{3} / \mathrm{SrTiO}_{3}$ heterojunction, Two-dimensional electron gas, Built-in electric field.

\begin{abstract}
The built-in electric field across $\mathrm{LaAlO}_{3} / \mathrm{SrTiO}_{3}$ heterointerface, which hosts two-dimensional electron gas (2DEG), is studied by off-axis electron holography in transmission electron microscopy (TEM). On the contrary to the well-accepted polar catastrophe model, which indicates a Zener breakdown due to the strong built-in electric field in $\mathrm{LaAlO}_{3}(\sim 0.24 \mathrm{~V} / \AA)$, we find that such an internal electric field in $\mathrm{LaAlO}_{3}$ is almost negligible $(<0.1 \mathrm{~V} / \AA$ A $)$ when compared to the band bending of $\mathrm{SrTiO}_{3}$. Our results suggest that the emergence of $2 \mathrm{DEG}$ should have other explanations beyond the polar catastrophe model.
\end{abstract}

\section{Introduction}

The conducting two-dimensional electron gas (2DEG) emerged at the heterointerface between two band insulators: $\mathrm{LaAlO}_{3}(\mathrm{LAO})$ and $\mathrm{SrTiO}_{3}$ (STO) has attracted intense research interest since its discovery by Ohtomo and Hwang [1]. Later on, a number of exotic physical properties have been found in such a model polar/nonpolar heterointerface system, e.g. superconductivity [2] and ferromagnetism [3]. However, the origin of the 2DEG still remains an open question. A well accepted interpretation for the conductivity was made based on the polar catastrophe model $[1,4,5]$, which postulates an intrinsic electronic reconstruction mechanism due to the polar discontinuity at the interface. According to electrostatics, the polar discontinuity between the nonpolar STO substrate and the polar LAO film would induce a strong built-in electric field across the interface. Particularly, the electrostatic potential in LAO increases divergently with increasing LAO thickness. As a result, at the critical thickness $d_{\mathrm{C}}$, a Zener breakdown will take place by means of charge transfer from the O $2 p$ valence band of LAO at the surface to the Ti $3 d$ conduction band of STO at the interface [7-9]. Based on this simple assumption by polar catastrophe model, the built-in electric field in LAO can be estimated to be about $\sim 0.24 \mathrm{~V} / \AA$. However, the presence of this built-in electric field has been questioned by numerous studies. For example, it is shown by X-ray photoemission [9-11] and scanning tunneling microscopy [12] that the internal field is only $\sim 0.02-0.03 \mathrm{~V} / \AA$, being much smaller than predictions by polar catastrophe model. Furthermore, it was found that $\mathrm{Ti}^{3+}$ signal appears well below $d_{\mathrm{C}}[11]$, which indicates a requirement of free charge density for coherent electron conduction. In order to explain these controversial experimental findings, especially the lack of strong built-in electric field in LAO, the oxygen vacancies model [13] and interface intermixing model [14] are proposed, respectively. Nevertheless, it is also questioned whether these hypotheses could explain the appearance of the critical thickness $d_{\mathrm{C}}$ for conductivity.

In this work, we focus on a direct measurement of the built-in electrostatic potential and electric field across the heterointerface by using electron holography. By comparing the built-in electric field in LAO film and that inside the substrate, it is shown that the built-in electric field in LAO is much smaller than the band bending in STO. Thus the built-in electric field should be $<0.1 \mathrm{~V} / \AA$ and cannot be simply explained by polar catastrophe model. The reason for such a small electric field is then discussed. 


\section{Experiments}

The cross-sectional 20 u.c. LAO/STO (001) sample studied in this paper was prepared in a conventional polishing and ion milling process [15]. Off-Axis Electron holography experiments were carried out on a FEI Titan 60-300 electron microscope equipped with a CEOS image aberration corrector and a bi-prism at Nanjing University. The microscope was operated at $300 \mathrm{kV}$ in transmission electron microscope (TEM) mode and the high-resolution electron holography images were recorded with a bias of $200 \mathrm{~V}$ applied on the bi-prism. The bi-prism was placed exactly parallel to the interface. In order to minimize the dynamical electron diffraction effect, the sample was tilted with respect to the normal of the interface, while the interface was kept edge on. The reconstruction of the amplitude $A(r)$ and phase $(r)$ of the coherent electron wave transmitted through the sample was performed by HoloWorks 5 plug-in for the software package Digital Micrograph. In principle, the phase change $(r)$ of the exit electron wave can be approximately related to the mean inner electrostatic potential $V(r)$ of the sample as

$$
\text { (r) } \quad C_{\mathrm{E}} V(r) t
$$

where $C_{\mathrm{E}}=E /$ is a constant that is related to the electron energy $E$ and wavelength ( $C_{\mathrm{E}}=6.52310{ }^{6} \mathrm{radV}^{-1} \mathrm{~nm}^{-1}$ for $300 \mathrm{kV}$ electrons) and $t$ is the sample's thickness. Therefore, we can estimate $V(r)$ and $E(r)=V^{\prime}(r)$ from $(r)$ straightforwardly if $(r)$ and $t$ are known. However, an accurate measurement of $t$ is generally complicated. As $t$ usually varies much less in a small region, we will thus only focus on the relative mean inner potential difference instead of the absolute mean inner potential. Ignoring the variation of thickness, Eq. 1 can be rewritten as

$$
\begin{gathered}
V(r) \mu \quad(r) \\
E(r)=V^{\prime}(r) \propto \quad{ }^{\prime}(r)
\end{gathered}
$$

Therefore, the internal electric field could be readily obtained once $(r)$ is reconstructed by electron holography.

\section{Results and Discussions}

Fig. 1 shows the recorded high-resolution electron holography and the corresponding Fast Fourier Transform (FFT) images, respectively. The interference fringes can be clearly observed with fringe contrast better than $10 \%$. The amplitude $A(r)$ and phase $(r)$ of the exit electron's wavefunction are reconstructed directly from one of the sideband with a resolution of $\sim 2.5 \mathrm{~nm}$, where the center of the sideband is indicated by the circle in the FFT image. Then the reconstructed phase $(r)$ is further processed by taking the vacuum as a reference and setting $(r)$ from the vacuum to zero. Fig. 2 displays the final reconstructed phase image $(r)$ [Fig. 2(a)] and the average line profile of (r) [Fig. 2(b)] across the interface, respectively. Obviously,

$(r)$ varies from the LAO film to the substrate in a continuous way. The width of this transition area is $\sim 2 \mathrm{~nm}$, being close to the width of cation intermixing interface [4]. Away from the interface, $(r)$ is constant in LAO and STO. Note that the phase change of LAO is larger than that of STO, indicating relatively larger mean inner potential of LAO. This result is consistent with the mean inner potential of bulk LAO and bulk STO, which is about $-25.9 \mathrm{~V}$ and $-22.3 \mathrm{~V}$ according to Refs. [16]. 

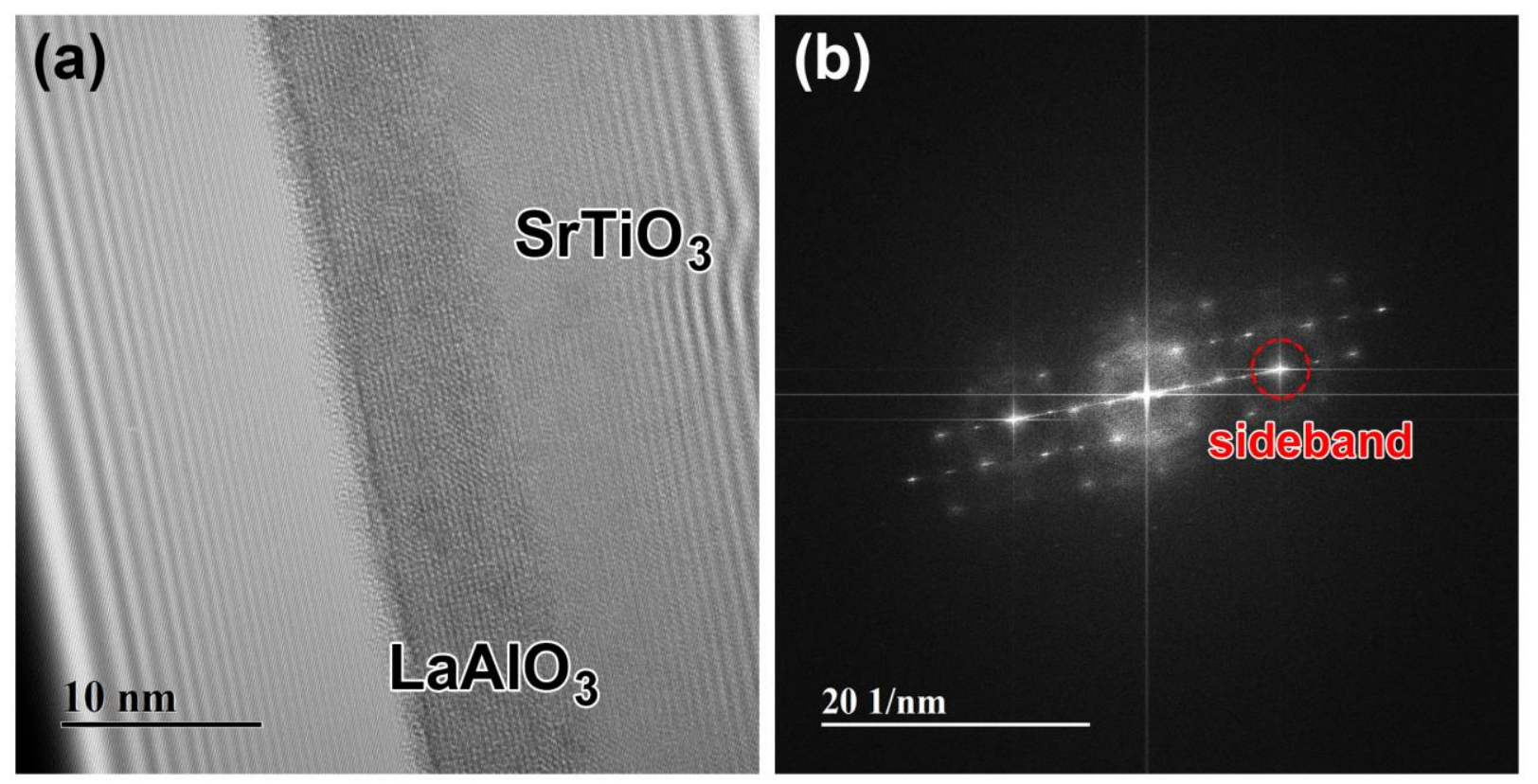

Fig. 1. (Color online) (a) Electron holographic image of $\mathrm{LaAlO}_{3} / \mathrm{SrTiO}_{3}(001)$ heterointerface. (b) Corresponding Fast Fourier Transform image of (a), where the sideband used for reconstruction is denoted by the red circle.

The relative built-in electric field $E$ is calculated from the derivative of $(r)$ profile and the result is shown in Fig. 2(b) as well. In accordance with the mean inner potential, the built-in electric field resides essentially in the interfacial region and the maximum is exactly at the interface. The negative sign of $E$ indicates that the electric field is pointing from the interface to the substrate. As a result, free charge carriers are attracted to the interface and form the 2DEG. Deep into the substrate, $E$ quickly decays to zero within $\sim 2 \mathrm{~nm}$. Note that study by scanning tunneling microscopy shows that the decay length of the band bending in STO is $\sim 0.8 \mathrm{~nm}$ [12]. The built-in electric field in LAO near the interface $(\sim 2 \mathrm{~nm})$ is negative as well, which probably is due to the cation intermixing of the interface [14]. In comparison, away from the interface, the built-in electric field in LAO slightly increases from zero to finite positive values, suggesting that the field is pointing from the interface to the surface and is in agreement with predictions by polar catastrophe model. Moreover, the relative field strength can be readily estimated from Fig. 2(b). As the electric field in STO is typically $\sim 0.1 \mathrm{~V} / \AA$ [ $[12,17]$, the field in LAO should be in the same scale or even less than $0.1 \mathrm{~V} / \AA$. This finding contradicts to the prediction by polar catastrophe model, but matches experiments $[11,12]$ very well. We remark that such controversy is not well understood within the framework of polar catastrophe model yet and it is either attributed to oxygen vacancies or interface intermixing. To shed light on the underlying mechanism, we phenomenologically expand the heterointerface's total energy as a function of the order parameter polarization and electric field using Landau theory. We found that the small built-in electric field can be immediately explained by the coupling between the polarization and electric field within LAO/STO heterointerface, which thus leads to the small inner potential and electric field. The details of the work will be reported elsewhere [18].

Note that at the surface of the film, the potential decreases to zero within a range of $\sim 3 \mathrm{~nm}$, which essentially corresponds to the unavoidable surface glue layer. This will give rise to a strong variation of the phase's derivative. In fact, if we consider the surface of a material, its mean inner potential will rapidly decrease to zero and give rise to a strong electric field localized at the surface. This is exactly what we have observed in Fig. 2. 

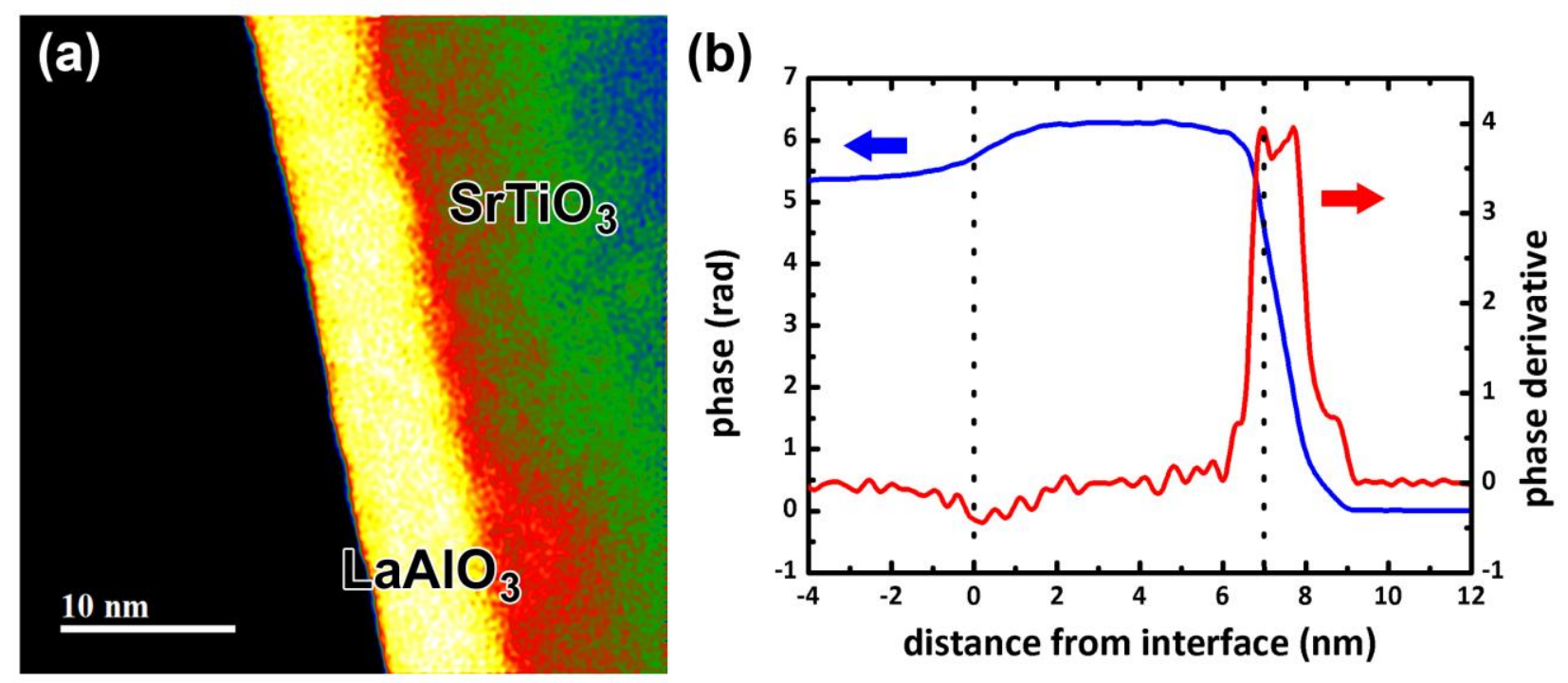

Fig. 2. (Color online) (a) Reconstructed phase

$(r)$ of the exit electron wave. (b) Profiles of the phase $(r)$ ( $\mu V(r))$ and phase derivatives $\quad '(r)(\mu E(r))$ across the heterointerface, where the interface and surface of the 20 u.c. $\mathrm{LaAlO}_{3}$ film is marked by the dashed lines.

\section{Summary}

We studied the built-in electric field in the 2DEG LAO/STO heterointerface by means of off-axis electron holography in a TEM. By reconstructing the phase of the exit electron wave that transmits through the heterointerface, we compared the relative strength of the built-in electric field in LAO and STO. It is found that the built-in electric field in LAO is much smaller than the band bending of STO and $<0.1 \mathrm{~V} / \AA$. Our results contradict the conventional understanding of the origin of $2 \mathrm{DEG}$, which is believed to be essentially caused by the strong built-in electric field within LAO. Hence, the emergence of 2DEG in LAO/STO heterointerface should have other explanations. Recently, our combined experiments and theoretical calculations based on Landau theory show that these controversies could be self-consistently explained if appropriate electrical boundary conditions are considered.

\section{Acknowledgement}

This research was financially supported by the National Natural Science Foundation of China (No. 51302132).

\section{References}

[1] A. Ohtomo, H.Y. Hwang, A high-mobility electron gas at the $\mathrm{LaAlO}_{3} / \mathrm{SrTiO}_{3}$ heterointerface, Nature. 427 (2004) 423-426.

[2] N. Reyren, S. Thiel, A.D. Caviglia, L.F. Kourkoutis, G. Hammerl, C. Richter, C.W. Schneider, T. Kopp, A.-S. Rüetschi, D. Jaccard, M. Gabay, D.A. Muller, J.-M. Triscone, J. Mannhart, Superconducting Interfaces Between Insulating Oxides, Science. 317 (2007) 1196-1199.

[3] J. A. Bert, B. Kalisky, C. Bell, M. Kim, Y. Hikita, H. Y. Hwang, K. A. Moler, Direct imaging of the coexistence of ferromagnetism and superconductivity at the $\mathrm{LaAlO}_{3} / \mathrm{SrTiO}_{3}$ interface, Nat. Phys. 7 (2011) 767-771.

[4] N. Nakagawa, H. Y. Hwang, D. A. Muller, Why some interfaces cannot be sharp, Nat. Mater. 5 (2006) 204-209. 
[5] C. Cancellieri, D. Fontaine, S. Gariglio, N. Reyren, A. D. Caviglia, A. Fête, S. J. Leake, S. A. Pauli, P. R. Willmott, M. Stengel, P. Ghosez, J.-M. Triscone, Electrostriction at the $\mathrm{LaAlO}_{3} / \mathrm{SrTiO}_{3}$ Interface, Phys. Rev. Lett. 107 (2011) 056102.

[6] S. A. Pauli, S. J. Leake, B. Delley, M. Björck, C. W. Schneider, C. M. Schlepütz, D. Martoccia, S. Paetel, J. Mannhart, P. R. Willmott, Evolution of the Interfacial Structure of $\mathrm{LaAlO}_{3}$ on $\mathrm{SrTiO}_{3}, \mathrm{Phys}_{\text {. Rev. }}$ Lett. 106 (2011) 036101.

[7] Z. S. Popovic, S. Satpathy, R. M. Martin, Origin of the Two-DimensionalElectron Gas Carrier Density at the $\mathrm{LaAlO}_{3}$ on $\mathrm{SrTiO}_{3}$ Interface, Phys. Rev. Lett. 101 (2008) 256801.

[8] J. Lee and A. A. Demkov, Charge origin and localization at the n-type $\mathrm{SrTiO}_{3} / \mathrm{LaAlO}_{3}$ interface, Phys. Rev. B. 78 (2008) 193104.

[9] Y. Segal, J. H. Ngai, J. W. Reiner, F. J. Walker, C. H. Ahn, X-ray photoemission studies of the metal-insulator transition in $\mathrm{LaAlO}_{3} / \mathrm{SrTiO}_{3}$ structures grown by molecular beam epitaxy, Phys. Rev. B. 80 (2009) 241107.

[10] M. Takizawa, S. Tsuda, T. Susaki, H. Y. Hwang, A. Fujimori, Electronic charges and electric potential at $\mathrm{LaAlO}_{3} / \mathrm{SrTiO}_{3}$ interfaces studied by core-level photoemission spectroscopy, Phys. Rev. B. 84 (2011) 245124.

[11] E. Slooten, Z. Zhong, H. J. A. Molegraaf, P. D. Eerkes, S. de Jong, F. Massee, E. van Heumen, M. K. Kruize, S. Wenderich, J. E. Kleibeuker, M. Gorgoi, H. Hilgenkamp, A. Brinkman, M. Huijben, G. Rijnders, D. H. A. Blank, G. Koster, P. J. Kelly, M. S. Golden, Hard x-ray photoemission and density functional theory study of the internal electric field in $\mathrm{SrTiO}_{3} / \mathrm{LaAlO}_{3}$ oxide heterostructures, Phys. Rev. B. 87 (2013) 085128.

[12] B.-C. Huang, Y.-P. Chiu, P.-C. Huang, W.-C. Wang, V. T. Tra, J.-C. Yang, Q. He, J.-Y. Lin, C.-S. Chang, Y.-H. Chu, Mapping Band Alignment across Complex Oxide Heterointerfaces, Phys. Rev. Lett. 109 (2012) 246807.

[13] A. Kalabukhov, R. Gunnarsson, J. Börjesson, E. Olsson, T. Claeson, D. Winkler, Effect of oxygen vacancies in the $\mathrm{SrTiO}_{3}$ substrate on the electrical properties of the $\mathrm{LaAlO}_{3} / \mathrm{SrTiO}_{3}$ interface, Phys. Rev. B. 75 (2007) 121404.

[14] L. Qiao, T. C. Droubay, T. C. Kaspar, P. V. Sushko, S. A. Chambers, Cation mixing, band offsets and electric fields at $\mathrm{LaAlO}_{3} / \mathrm{SrTiO}_{3}(001)$ heterojunctions with variable $\mathrm{La}$ :Al atom ratio, Surf. Sci. 605 (2011) 1381-1387.

[15] D.B. Williams, C.B. Carter, Transmission Electron Microscopy: A Textbook for Materials Science, Springer US, 2009.

[16] E.J. Kirkland, Advanced Computing in Electron Microscopy, 2nd ed., Springer US, 2010.

[17] O. Copie, V. Garcia, C. Bödefeld, C. Carrétéro, M. Bibes, G. Herranz, E. Jacquet, J.-L. Maurice, B. Vinter, S. Fusil, K. Bouzehouane, H. Jaffrès, A. Barthélémy, Towards Two-Dimensional Metallic Behavior at $\mathrm{LaAlO}_{3} / \mathrm{SrTiO}_{3}$ Interfaces, Phys. Rev. Lett. 102 (2009) 216804.

[18] L. Xie, S. Wang, C.-B. Eom, P. Wang, X. Pan, Induced polarization and its role in the two-dimensional electron gas at the polar/nonpolar $\mathrm{LaAlO}_{3} / \mathrm{SrTiO}_{3}$ interface, unpublished. 